\title{
RADIATIVE LIFETIMES AND COLLISION PROCESSES IN SODIUM-RARE GAS AND SODIUM-HYDROGEN SYSTEMS
}

\author{
L. H. VASSIE \\ Department of Mechanical Engineering, Loughborough University of Technology, \\ Loughborough, Leics. LEII $3 T U$
}

H. H. TELLE

Department of Physics, University College of Wales, Swansea, Singleton Park. SA2 8PP. Wales.

(Received 8 March, 1993)

\begin{abstract}
Investigations of the collisional interactions between sodium atoms in the $3^{2} \mathrm{P}$ states and helium and hydrogen gases are described. Studies of collision-induced mixing and quenching interactions have been carried out under conditions where chemical reaction to produce sodium hydride is possible. Thus the sodium number derivatives are sufficient for radiation trapping to occur. This is in contrast to virtually all previous investigations in which conditions were such that this effect need not be considered. The intensity and temporal behaviour of laser induced fluorescence (LIF) resulting from the photoexcitation of the sodium $3^{2} \mathrm{P}$ states is monitored as a function of foreign gas pressure. Whilst many of the features observed are to be expected, results show how radiation trapping strongly influences cross-section determination. The need for a more rigorous treatment of collision cross-sections under these conditions, which are often more realistic than the 'ideal' trapping free situations, is demonstrated.
\end{abstract}

KEY WORDS: Sodium, Hydrogen, Rare-gas, Collisions, Radiation Trapping, Laser Induced Fluorescence.

\section{INTRODUCTION}

Collisions of alkali metal atoms with a number of inert gases and diatomic molecules has been the subject of numerous experimental and theoretical investigations to obtain information on processes such as quenching cross-sections and collision-induced mixing in the $3^{2} \mathrm{P}$ levels. In virtually all of these investigations the conditions are such that the phenomena of radiation trapping need not be considered. This simplifies the rate equations used for cross-section determination.

Previous investigations of the collisions of excited alkali atoms $\left(3^{2} \mathrm{P}\right)$ with inert gases and diatomic gases have focused on intensity measurements of the fluorescence emanating from these excited states as a function of foreign gas pressure. All inves- 
tigations are long standing and there have been no new investigations. Early studies of $\mathrm{Na} \mathrm{D}$ line fluorescence ${ }^{1,2}$ showed the influence of radiation trapping; the ratio of the intensities of the two D line components did not approach $2: 1$ (as predicted by principle of detailed balancing). Collision-induced mixing resulting from the collisions of excited alkali atoms and rare gas atoms, has been studied in some detail, however, under radiation free trapping conditions and cross-sections produced. ${ }^{3-5}$ Quenching of the $\mathrm{Na} \mathrm{D}$ line radiation by a number of gases have been studied via various methods, which essentially vary in the way in which the excited species are produced. ${ }^{4-11}$ All, with the exception of Demtroder, ${ }^{8}$ measured the variation of fluorescence intensity with foreign gas pressure. Demtroder used the variation of the lifetime of the excited state with gas pressure, the significance of which will be discussed later. Jenkins ${ }^{6}$ made allowances for radiation trapping, however, the excited states were studied by flame fluorescence methods.

Investigations of radiation trapping have, as with the other collision processes described above, been analysed in isolation with no other effects present. Studies of the effects of imprisonment of radiation for the $\mathrm{Na} 3^{2} \mathrm{P}$ states have shown a difference in lifetimes of the two $\mathrm{Na} \mathrm{D}$ line components at high sodium vapour pressures. ${ }^{12}$

The investigations described herein present the experimental results achieved from studies of the intensity and temporal behaviour of laser induced $\mathrm{Na} \mathrm{D}$ line fluorescence under conditions where the process of radiation trapping, quenching or collision induced mixing occur simultaneously.

\section{COLLISION PROCESS IN Na-H $\mathrm{H}_{2}$ AND Na-He MIXTURES}

Consider the collision of a sodium atom in its ground $\mathrm{Na}$ or excited state $\mathrm{Na}^{*}$ with a helium or hydrogen. The following processes are possible:

1. Collisional transfer of energy from $\mathrm{Na}^{*}$ to $\mathrm{He}$ or $\mathrm{H}_{2}$ :

or

$$
\begin{aligned}
& \mathrm{Na}^{*}+\mathrm{He} \rightarrow \mathrm{Na}+\mathrm{He}^{*} \\
& \mathrm{Na}^{*}+\mathrm{H}_{2} \rightarrow \mathrm{Na}+\left(\mathrm{H}_{2}\right)^{*}
\end{aligned}
$$

2. Inter-level energy transfer from excited state $\mathrm{Na}^{*}$ to excited state $\mathrm{Na}^{* *}$ :

or

$$
\begin{aligned}
& \mathrm{Na}^{*}+\mathrm{He} \rightarrow \mathrm{Na}^{* *}+\mathrm{He} \\
& \mathrm{Na}^{*}+\mathrm{H}_{2} \rightarrow \mathrm{Na}^{* *}+\mathrm{H}_{2}
\end{aligned}
$$

3. Chemical reaction:

or

$$
\begin{aligned}
& \mathrm{Na}+\mathrm{H}_{2} \rightarrow \mathrm{NaH}+\mathrm{H} \\
& \mathrm{Na}^{*}+\mathrm{H}_{2} \rightarrow \mathrm{NaH}^{*}+\mathrm{H}
\end{aligned}
$$

When investigating reaction dynamics the primary interest is to ascertain the efficiency of the possible processes. A measure of this efficiency factor is the cross-section. 
In the gas mixture reactions (1), (2) and (3) will occur with various efficiencies and thus cross-sections depending on the conditions. In order to establish the crosssections for processes (1) and (2) it is usual to do so with a small density of A in order to avoid the problems associated with radiation trapping which makes direct quantitative measurement of either difficult or impossible as has been observed. ${ }^{9,10}$ On the other hand, in order to establish a cross-section for the reaction process (3) a large number density of $\mathrm{A}$ is necessary to produce a sufficient amount of the product molecule for analysis. Experimental determination of collision cross-sections for the processes (1) and (2) can be reached via measurements of both the variation of the fluorescence intensity and its temporal behaviour with the concentration of the collision partner.

\section{Process 1}

When a foreign gas is added to excited species emitting a fluorescing vapour the intensity of the fluorescence will often be diminished by an amount depending on both the pressure and nature of the gas. The fluorescence intensity is said to be quenched and the collision in which this occurs is a quenching collision. The energy of the excited particle is transferred partially or wholly to kinetic or excitation energy of the gas particle. The 'quenching' $Q$ is defined as the ratio of fluorescence intensity with the gas present to that in its absence, i.e. $Q=I_{1} / I_{0}$ where $I_{1}$ and $I_{0}$ are the intensities of fluorescence with and without the gas present. The behaviour of the ratio $I_{1} / I_{0}$ with gas pressure is usually described by the Stern-Volmer relation:

$$
\mathrm{I}_{0} / \mathrm{I}_{1}=1+\tau \mathrm{Z}_{\mathrm{Q}}
$$

where $Z_{Q}$ is the number of quenching collisions made per second per excited atom and $\tau$ is the lifetime of the excited state. The relationship may also be expressed as:

$$
\mathrm{I}_{0} / \mathrm{I}_{1}=1+\left(\sigma \mathrm{p} \tau_{0}\right)(8 / \pi \mathrm{mkT})^{1 / 2}
$$

since $\mathrm{Z}_{\mathrm{Q}}=\mathrm{N} \sigma \mathrm{v}_{\mathrm{r}}$ where $\mathrm{N}$ is the number of foreign gas molecules per $\mathrm{cm}^{-3}, \sigma$ is the quenching cross-section and $\mathrm{v}_{\mathrm{r}}$ is the relative velocity of the colliding particles and $\mathrm{N}=\mathrm{pkT}$.

The effective cross-section for quenching may be determined from the slope of the $I_{0} / I_{1}$ vs pressure plot if the average lifetime $\tau_{0}$ is known from other experiments.

The Stern-Volmer relationship can also be expressed in terms of the lifetimes of the excited state with and without a quenching gas present:

$$
\mathrm{I}_{0} / \mathrm{I}_{1}=1+\tau_{0} / \tau_{1}
$$

where $\tau_{1}$ is the average time for the excited particle to make a quenching collision i.e. $\tau_{1}=1 / \mathrm{Z}_{\mathrm{Q}}$.

It should be pointed out that application of the Stern-Volmer relation assumes that (i) the absorbing gas pressure is sufficiently low that resonance radiation is not further absorbed and (ii) the foreign gas pressure is sufficiently low that Lorentz broadening of the absorption line is negligibly small. However, in many cases, where these criteria are not satisfied, a linear variation of $\mathrm{I}_{0} / \mathrm{I}$ (i.e. $1 / \mathrm{Q}$ ) with pressure is produced. 
In fact almost any portion of a descending curve can be fitted to a Stern-Volmer type formula. If the same quenching curve is obtained at various low absorbing gas pressures, then the region in which the formula is applicable has been obtained.

\section{Process 2}

When an alkali-foreign gas mixture is irradiated with light of wavelength corresponding to that of one component of the resonance doublet both components appear in fluorescent light, one as "resonance fluorescence", the other as "sensitised fluorescence". The latter is the result of inelastic collisions between excited state alkali atoms and the foreign gas atoms. This process is known as collision-induced mixing.

For the case in which collision-induced mixing amongst the fine structure levels is the only process occurring in addition to excitation of resonant level and its spontaneous decay, then conditions in the alkali-gas mixture can be represented by two pairs of rate equations each pair corresponding to excitations with one D-line component. If the ${ }^{2} \mathrm{P}_{\frac{1}{2}}$ level is excited the pair of equations is:

$$
\begin{gathered}
\mathrm{dN}_{1} / \mathrm{dt}=-\left(1 / \tau_{1}\right) \mathrm{N}_{1}-\mathrm{Z}_{1} \mathrm{~N}_{1}+\mathrm{Z}_{2} \mathrm{~N}_{2}+\mathrm{S}_{1}=0 \\
\mathrm{dN}_{2} / \mathrm{dt}=-\left(1 / \tau_{2}\right) \mathrm{N}_{2}-\mathrm{Z}_{1} \mathrm{~N}_{1}+\mathrm{Z}_{2} \mathrm{~N}_{2}=0
\end{gathered}
$$

where $N_{1}$ is the number density of the ${ }^{2} \mathrm{P}_{\frac{1}{2}}$ state, $\mathrm{N}_{2}$ is the number density of the collisionally excited ${ }^{2} \mathrm{P}_{\frac{3}{2}}$ atoms, $\mathrm{Z}_{1}$ and $\mathrm{Z}_{2}$ are the collision frequencies per excited atom corresponding to the processes ${ }^{2} \mathrm{P}_{\frac{1}{2}} \rightarrow{ }^{2} \mathrm{P}_{\frac{3}{2}}$ and ${ }^{2} \mathrm{P}_{\frac{3}{2}} \rightarrow{ }^{2} \mathrm{P}_{\frac{1}{2}}$ respectively, $\mathrm{S}$ is the number density of atoms excited per second to the ${ }^{2} \mathrm{P}_{\frac{1}{2}}$ state by the incident radiation, and $\tau_{1}$ and $\tau_{2}$ are the lifetimes of the two resonance states. These equations, together with a similar set corresponding to excitations of the ${ }^{2} \mathrm{P}_{\frac{3}{2}}$ level, may be solved to give expressions for $Z_{1}$ and $Z_{2}$ :

$$
\begin{aligned}
& \mathrm{Z}_{1}=\left(1 / \tau_{1}\right)\left[1+\eta_{1}\right] /\left[\left(1 / \eta_{2}\right)-\eta_{1}\right] \\
& \mathrm{Z}_{2}=\left(1 / \tau_{2}\right)\left[1+\eta_{2}\right] /\left[\left(1 / \eta_{1}\right)-\eta_{2}\right]
\end{aligned}
$$

where $\eta_{1}$ and $\eta_{2}$ are the ratios of intensities of sensitised-to-resonance fluorescence, i.e.

$$
\eta_{1}=\mathrm{I}_{1}\left(\mathrm{D}_{1}\right) / \mathrm{I}_{2}\left(\mathrm{D}_{2}\right): \eta_{2}=\mathrm{I}_{2}\left(\mathrm{D}_{2}\right) / \mathrm{I}_{1}\left(\mathrm{D}_{1}\right)
$$

The collision cross-sections $\mathrm{Q}_{1}$ and $\mathrm{Q}_{2}$ for the two excitation transfer processes may be obtained from the definition of the gas-kinetic cross-section:

$$
Z_{1,2}=N Q_{1,2} v_{r}
$$

where $\mathrm{N}$ is the number density of the foreign gas atoms and $\mathrm{v}_{\mathrm{r}}$ is the average relative velocity of the colliding atoms $\left(v_{r}=\sqrt{ }(8 \mathrm{kT} / \pi \mathrm{m}) ; \mathrm{m}=\mathrm{m}_{1} \mathrm{~m}_{2} / \mathrm{m}_{1}+\mathrm{m}_{2}\right)$. These equa- 
tions, (8.1) and (8.2), make no allowance for the processes of quenching and radiation trapping. In previous studies of collisional excitation transfers in alkali-inert gas systems quenching has been assumed negligible and radiation trapping is ineffective at the alkali number densities used i.e. below $-3 \times 10^{12} \mathrm{~cm}^{-2}$.

\section{Radiation Trapping}

When the density of the species under investigation is sufficiently high there is an appreciable probability that a photon emitted by an excited atom may subsequently be absorbed and re-emitted by other unexcited atoms in the system before 'escaping' to be detected. This 'radiation trapping' process effectively 'delays' the photon and results in the lifetime appearing to be much longer than the natural lifetime.

Several re-absorptions and re-emissions may occur before the radiation escapes, and thus the process may be seen as being similar to that of diffusion. The effect is dependent on the density of absorbing atoms. The diffusion of resonance radiation is well documented ${ }^{13-15}$ and these have produced several mathematical representations of the process. Essentially the effect results in a transition probability $A_{n o}$ being replaced by a reduced probability $A_{n o}^{\prime}$ where $A_{n o}^{\prime}=(1-x) A_{n o}$ resulting in an apparently extended lifetime $\tau^{\prime}=\tau /(1-x)$. The parameter $x$ is a functional integral of time, dependent on absorber density $\rho$ and a characteristic absorption length. Milne's treatment ${ }^{16}$ yields an expression of the form

$$
\tau^{\prime}=\tau\left[1+(\mathrm{C} / \lambda)^{2}\right]
$$

The quantity $\mathrm{C}$ is the opacity of the absorbing vapour and can be expressed as $\mathrm{C}=$ $\alpha \mathrm{lN}=\mathrm{kl}$ where $\alpha$ is the absorption cross-section for the resonance line in question, $\mathrm{N}$ is the number density, 1 is the thickness of the vapour layer responsible for the diffusion of radiation, and $\mathrm{k}$ is the equivalent absorption coefficient. ${ }^{17} \lambda$ is given $\mathrm{t}$ $\lambda \tan \lambda=\mathrm{C}$.

\section{EXPERIMENTAL}

The apparatus used is illustrated schematically in Figure 1. It essentially comprises three main components: (i) the alkali-gas interaction system; (ii) the laser excitation; (iii) the fluorescence detection.

\section{Alkali-gas interaction system}

The alkali-gas interaction system is an all glass system consisting of an alkali oven in which the sodium is electrically heated to produce a sodium number density of $\sim 10^{14} \mathrm{~cm}^{-3}$. Vapour effuses into the main tube and is carried along the tube by the coaxial $\mathrm{H}_{2} / \mathrm{He}$ gas flow. Excitation of the $\mathrm{Na}-\mathrm{H}_{2}$ mixture in order to create the conditions for $\mathrm{NaH}$ formation, which was a further objective of the investigation, is provided by either a microwave or RF discharge. 


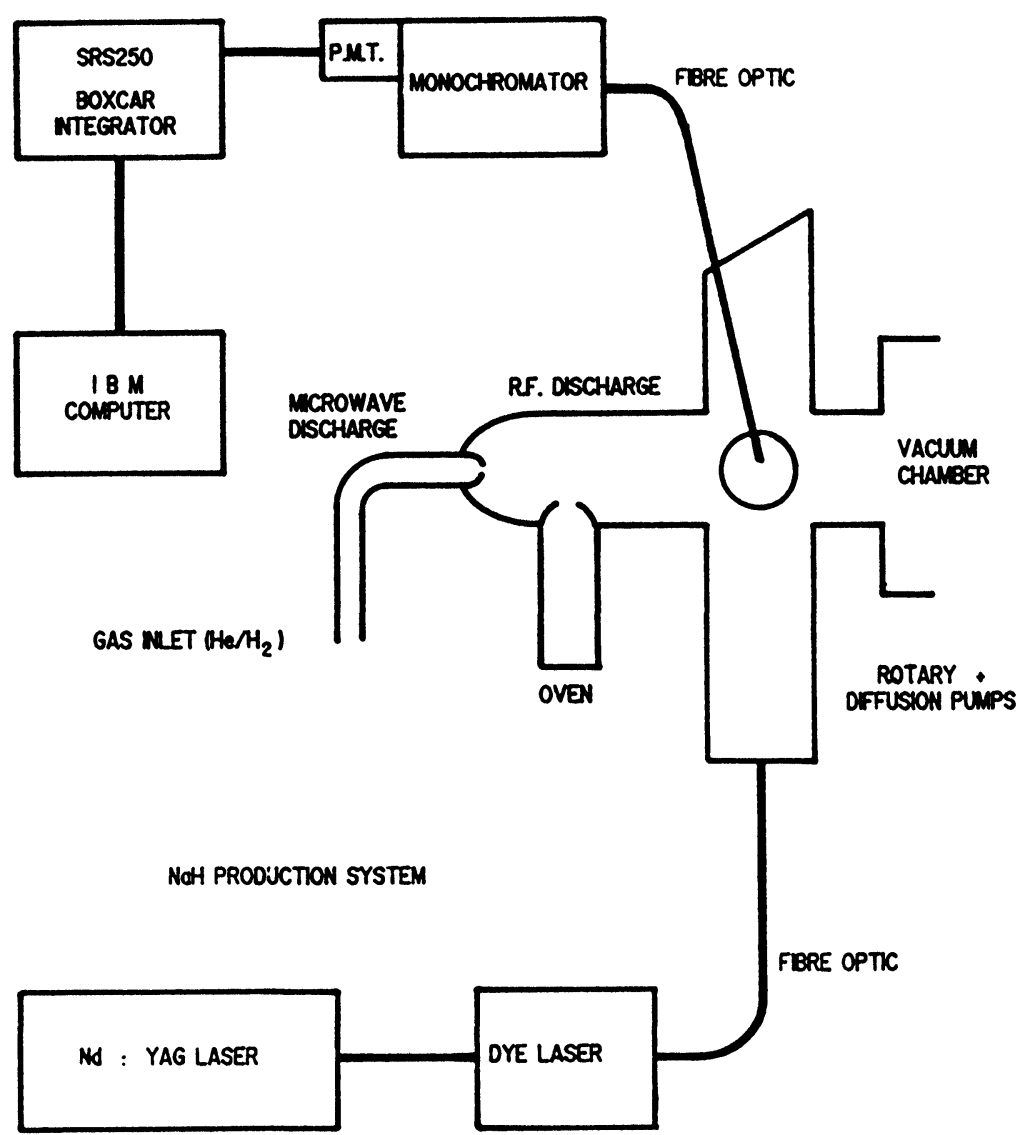

Figure 1 Schematic diagram of experimental set up

Both $\mathrm{H}_{2}$ and sodium enter the main flow tube via tapered nozzles. These prevent (1) sodium vapour diffusing into the gas inlet and (2) sudden surges of sodium vapour into the flow tube. The complete reaction zone is heated by a thermocoax coil to temperatures in the range $300-350^{\circ} \mathrm{C}$, giving a sodium vapour pressure of $0.05-0.24$ mbar, to prevent condensation of sodium. The reaction system is shown in Figure 2.

A few centimetres downstream from the reaction region, access is provided for the laser excitation. The laser beam enters via a fused silica plane window, passes through the excitation region and exits via another window mounted at the Brewster angle. A Wood's Horn is situated on the exit tube such that any light reflected back from the exit window will be directed into it and become trapped. A short tubulation above the excitation zone, mutually perpendicular to the flow tube and laser beam supports a fused silica lens to collect the fluorescence from the excitation region. The side arms are heated to prevent sodium condensation. 


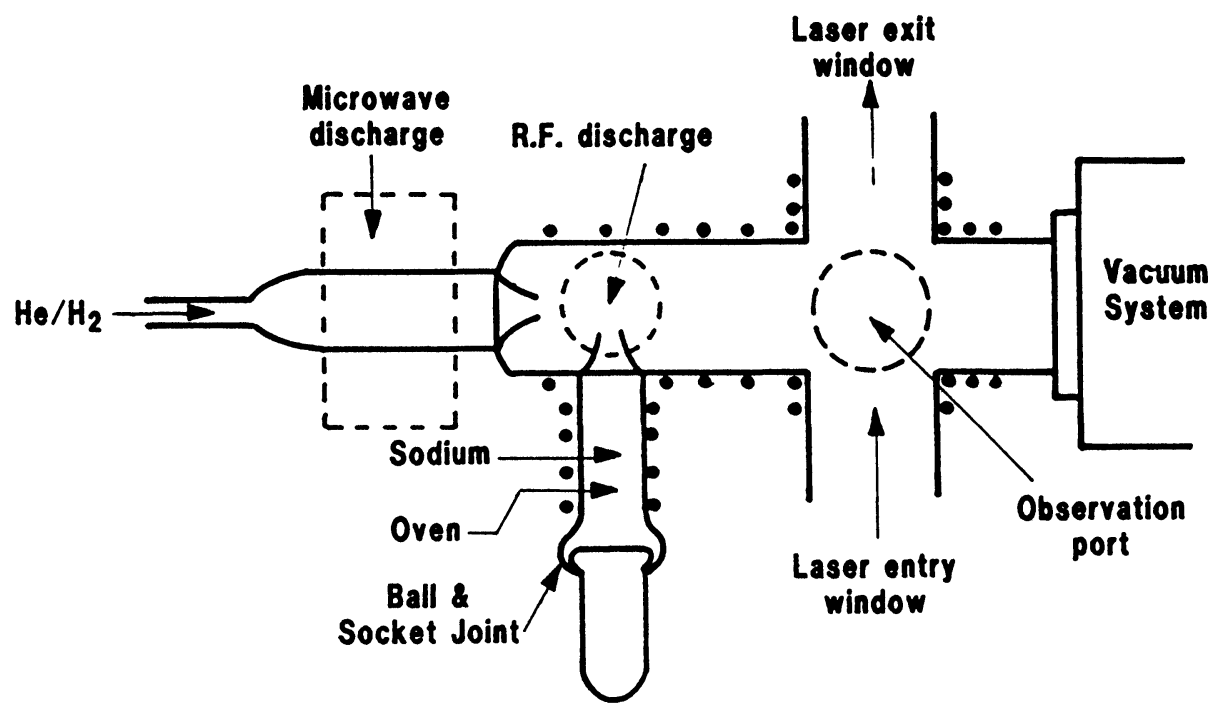

Figure $2 \mathrm{Na}-\mathrm{H}_{2}$ reaction system

The flow region and excitation region are evacuated to $\sim 10^{-4}$ mbar prior to the reaction process using a two stage rotary and oil diffusion pump system. Flow of gases into the system is controlled by vernier valves.

\section{Laser Excitation}

This is provided by an Nd:YAG pumped grazing incidence dye laser. In these investigations the frequency doubled output of the Nd:YAG laser at $532 \mathrm{~nm}$ is used to excite the Rhodamine $\mathrm{B}$ dye in the dye laser. The output from the pulsed dye laser has an approximate line width of $0.1 \AA$; the output is tunable to $\pm 1 \AA$ over the tuning range of the dye $(570-605 \mathrm{~nm})$. The energy per pulse is approximately $1 \mathrm{~mJ}$.

Fibre optic delivery systems are used for transporting the dye laser beam to, and the fluorescence from the excitation region.

\section{Detection System}

Fluorescence emitted as a result of the photoexcitation of the $\mathrm{Na}-\mathrm{H}_{2}$ and $\mathrm{Na}-\mathrm{He}$ mixture is spectrally analysed by a grating monochromator (Bentham M300) and its intensity measured by a photomultiplier tube (Thorn EMI 9816Q13). The signal from the photomultiplier tube is recorded by a boxcar integrator (Stanford SR250) interfaced to an IBM PC. A data acquisition package (SR 265:EXE) is used to record and analyse the data. The data acquisition process is activated by a pulse from an external trigger unit whose input is obtained from a PIN diode situated at a port in the Nd:YAG laser prism harmonic separator unit (PHS); a back reflection from the fundamental beam produces a current pulse in the PIN diode. 


\section{Experimental Procedures}

Two sets of experiments were carried out in which the sodium-helium or sodium hydrogen mixture was photo-excited with radiation from the dye laser at $589.0 \mathrm{~nm}$ or $589.6 \mathrm{~nm}$ coinciding with the $\mathrm{Na} 3^{2} \mathrm{~S} \rightarrow 3^{2} \mathrm{P}_{\frac{3}{2}}$ and $3^{2} \mathrm{~S} \rightarrow 3^{2} \mathrm{P}_{\frac{1}{2}}$ transitions respectively. In the first set of experiments the intensity of the laser induced fluorescence at $589.0 \mathrm{~nm}$ and $589.6 \mathrm{~nm}$ was monitored as a function of helium and hydrogen gas pressures in the range 0 to 25 mbar. The monochromator was scanned through a $4 \mathrm{~nm}$ range from $588 \mathrm{~nm}$ to $592 \mathrm{~nm}$ in the presence and absence of the RF discharge. Additionally the $4 \mathrm{~nm}$ wavelength scan was performed in the absence of sodium vapour, with only a low background gas pressure $(<1.0 \mathrm{mbar})$ in order to determine the scattered light levels in the system. This signal is subsequently subtracted from the other wavelength scans. The dye laser power was also recorded for each wavelength scan to enable the fluorescence signals to be normalised to the laser power.

For each excitation wavelength $(589.0 \mathrm{~nm}$ or $589.6 \mathrm{~nm})$ the variation of the resonance and sensitised fluorescence intensities and the ratio of sensitised to resonance intensities was investigated as a function of gas pressure.

In the second set of experiments the temporal behaviour of the laser-induced fluorescence was monitored as a function of the gas pressure in the same range as above. The boxcar gate, $3 \mathrm{~ns}$ width, is scanned through the fluorescence signal. The gate is scanned through a width of $600 \mathrm{~ns}$ with 30 samples being taken at each gate position.

In addition, the temporal behaviour of the scattered light at both observation wavelengths, in the absence of sodium vapour, was recorded. The scattered light signal must be subtracted from the time scans recorded in the presence of sodium since these scans, having no preset delay prior to the boxcar gate opening, have contributions from scattered light and the fluorescence. Lifetimes are extracted from the decay curves using the data reduction routines available with the boxcar program.

\section{RESULTS}

\section{Intensity Measurements}

With increasing foreign gas pressure the rate of transfer of excited atoms between the two states is expected to reach a steady state. This state is indicated by the variation of $\eta_{1}$ and $\eta_{2}$ with pressure. According to the Principle of Detailed Balancing predicted values of $\eta_{1}$ and $\eta_{2}$ are 0.53 and 1.88 respectively. These experiments indicate values of $\eta_{1}$ and $\eta_{2}$ of 0.45 and 1.4 respectively at a helium pressure of 25 mbar (Figure 3). Comparison with the work of Pitre ${ }^{5}$ shows that $\eta_{2}$ is in good agreement at a similar pressure, however, there is a discrepancy with the value of $\eta_{1}$. This can be attributed to the effect of radiation trapping; the probability of trapping the $589.0 \mathrm{~nm}\left(3^{2} \mathrm{P}_{\frac{3}{2}}\right)$ radiation is twice that for the $589.6 \mathrm{~nm}\left(3^{2} \mathrm{P}_{\frac{1}{2}}\right)$ radiation. The variation of fluorescence intensity with sodium vapour pressure is expected to be 


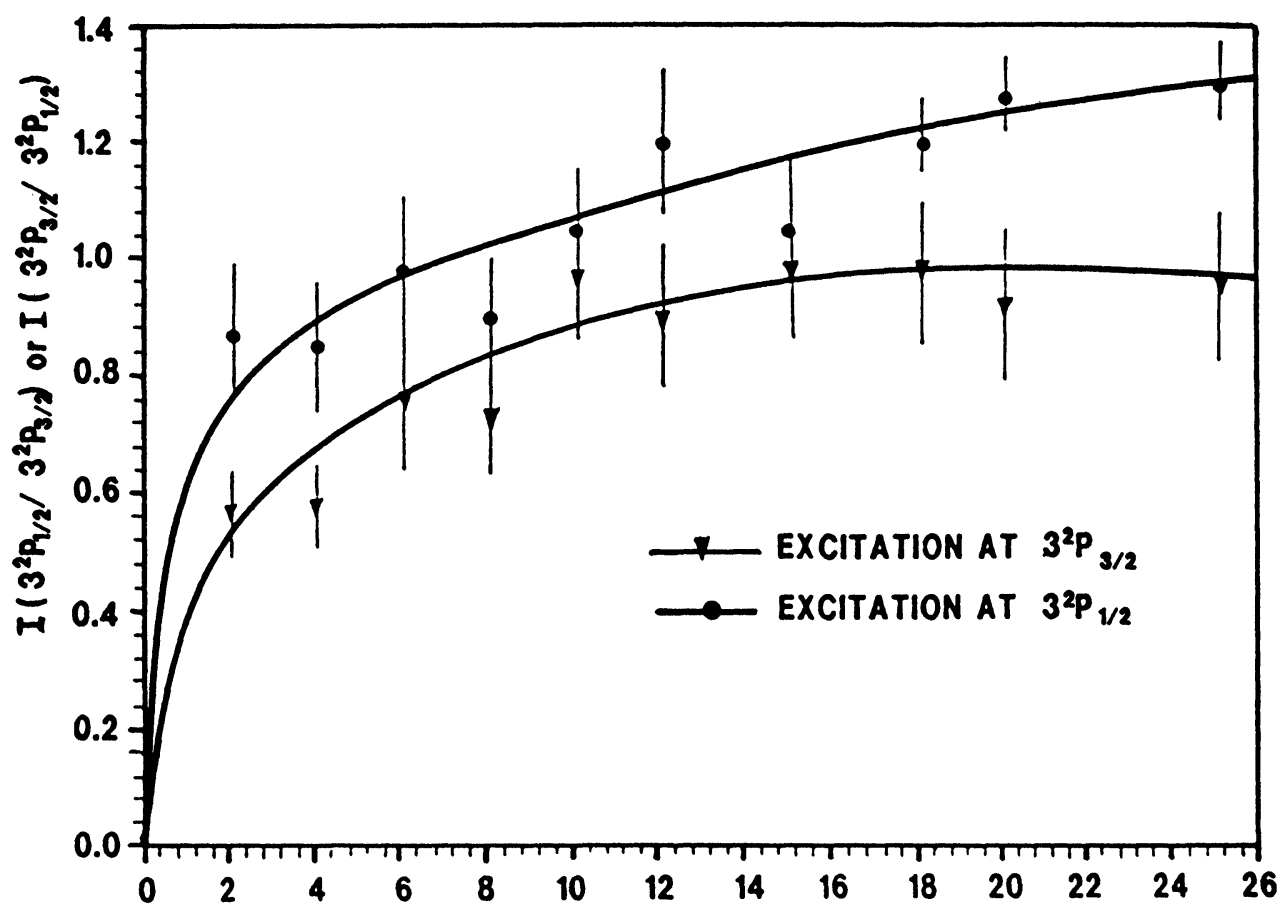

Figure 3 Ratio of sensitised to resonance $\mathrm{Na}$ fluorescence intensities versus helium pressure

linear at low vapour pressures and depart from linearity when trapping sets in; the vapour pressure at which this occurs for the radiation from the $3^{2} \mathrm{P}_{\frac{3}{2}}$ level is approximately half that at which it occurs for the radiation from the $3^{2} \mathrm{P}_{\frac{1}{2}}$ level. In a similar way the ratios $\eta_{1}$ and $\eta_{2}$ should be linear with vapour pressure until radiation trapping sets in, at which point they increase rapidly and non-linearly. It appears in these experiments that $\eta_{1}$ is in this non linear region.

Total intensity measurements i.e. resonance and sensitised fluorescence, in the presence of helium are in excess of the intensity at zero foreign gas pressure (Figure 4). This effect could be attributed to collisions between $\mathrm{Na}^{*}$ and helium atoms, allowing resonance radiation trapped at zero foreign gas pressure to escape. This effect was not observed in hydrogen, which exhibited the expected stronger quenching effects (Figure 5). The total fluorescence intensity decreases by $80 \%$ over the 25 mbar range and has only a very small contribution from the collisional transfer. If a quenching curve (Figure 6) and subsequently a 1/Q curve vs pressure is plotted, the gradient of the line $\left(\sigma \tau(8 / \pi \mathrm{mkT})^{1 / 2}\right)$ yields cross-section values of $35 \times 10^{-16} \mathrm{~cm}^{2}$ and $35 \times 10^{-16} \mathrm{~cm}^{2}$ for excitation at $3^{2} \mathrm{P}_{\frac{1}{2}}$ and $3^{2} \mathrm{P}_{\frac{3}{2}}$ respectively.

When the sodium-helium mixture is subjected to the R.F discharge prior to laser excitation a stronger quenching effect is observed. The loss of intensity suggests that 


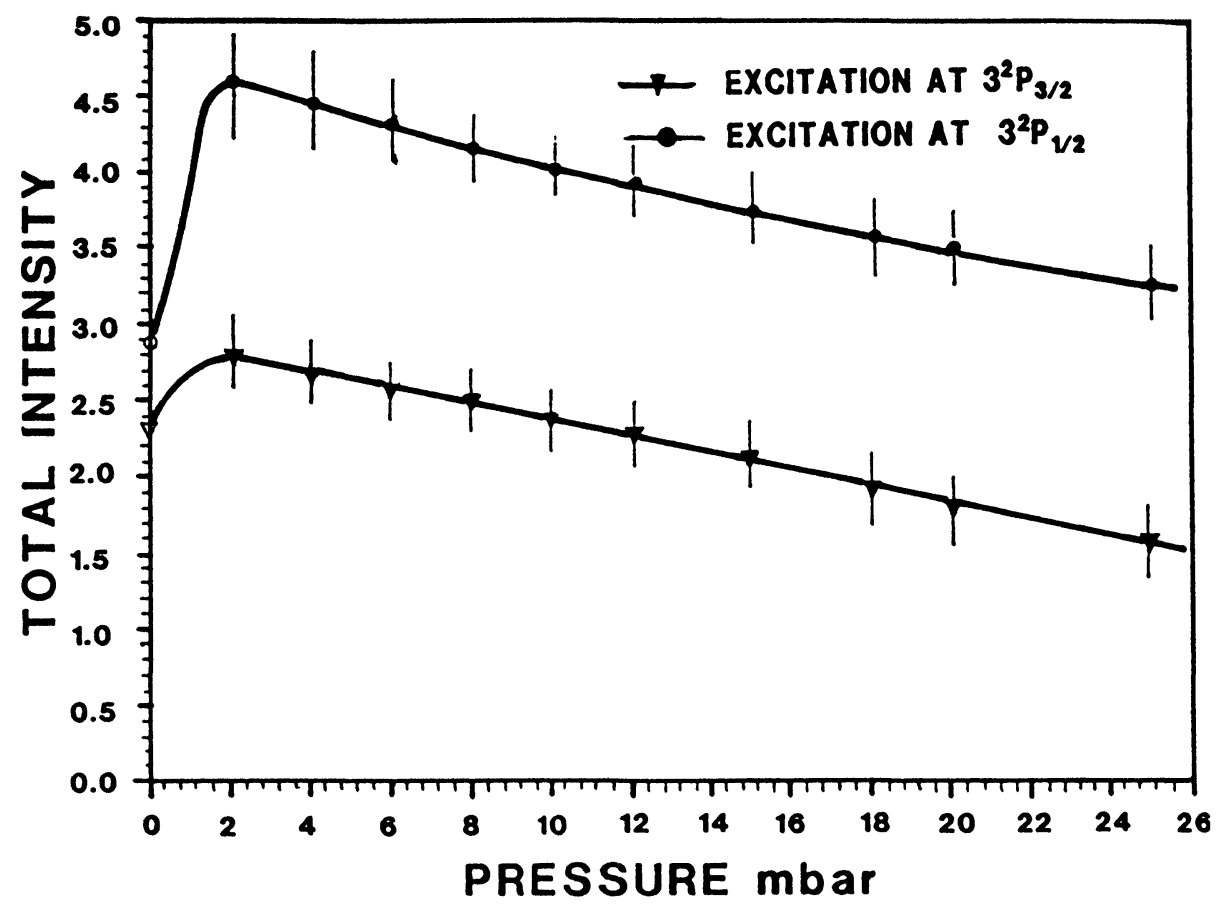

Figure 4 Total $\mathrm{Na}$ fluorescence intensities versus helium pressure

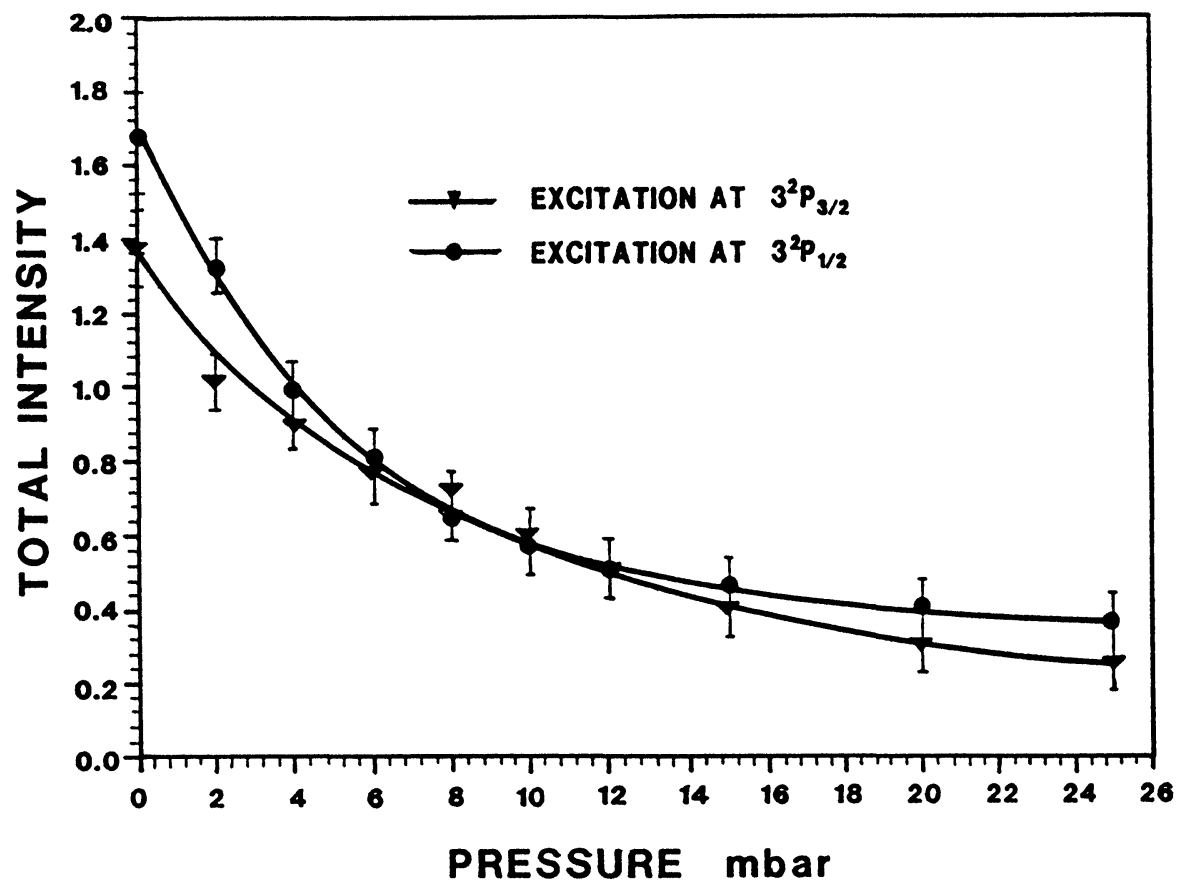

Figure 5 Total Na fluorescence intensities vercıs hydrogen pressure 


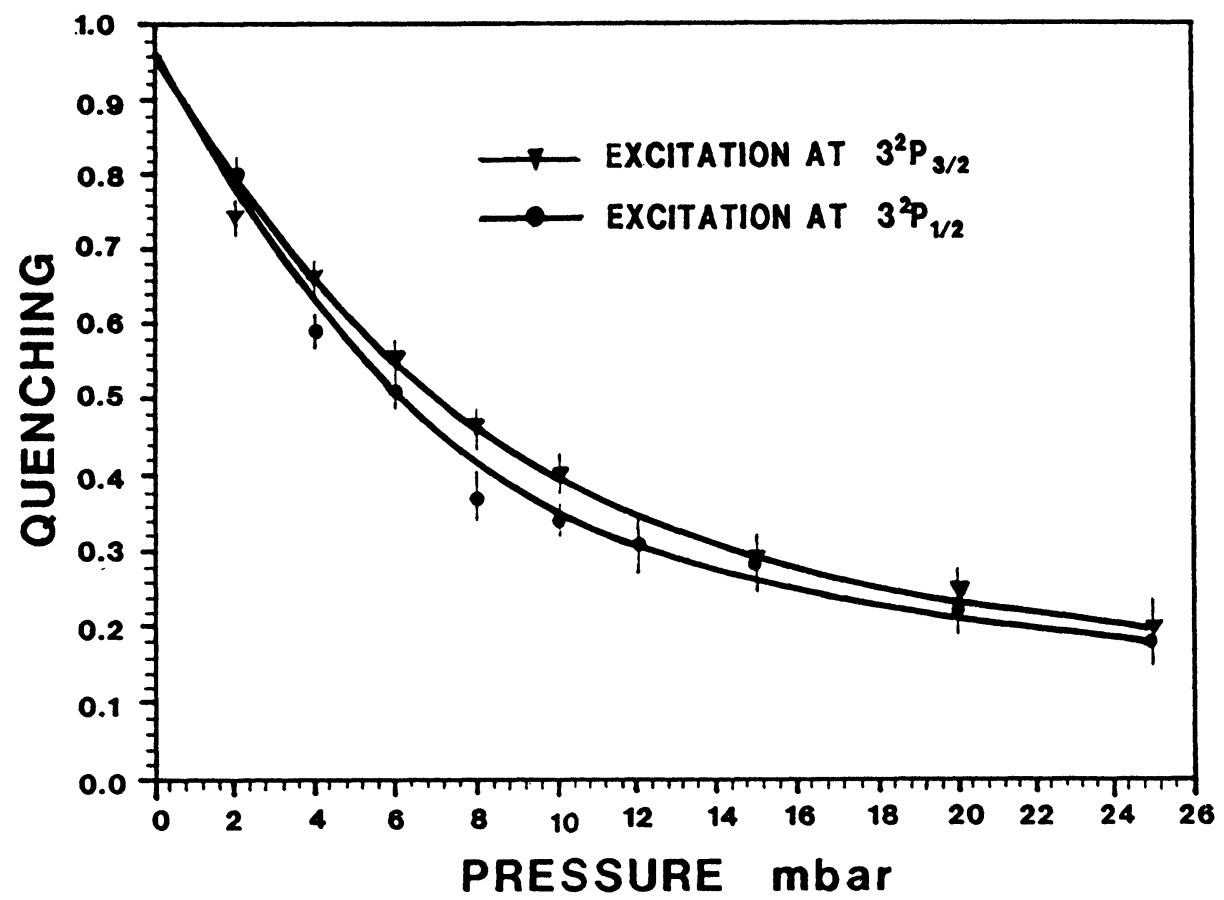

Figure 6 Quenching curves for total Na fluorescence versus hydrogen pressure

excited helium species interact in different way to their unexcited counterparts and introduce a quenching effect. In the discharge helium is produced in a number of excited states, and in particular the metastable $2^{3} S_{1}$ and $2^{1} S_{0}$ states are populated. Due to their long lifetimes there is an increased probability of collisions with $\mathrm{Na}$ $3^{2} \mathrm{P}$ states. Furthermore, the energy of these excited states is $\sim 23 \mathrm{eV}$ and thus transfer of energy from $\mathrm{Na} 3^{2} \mathrm{P}$ having an energy of $2.1 \mathrm{eV}$ would result in ionised helium $\left(\mathrm{E}_{\mathrm{ion}}=24.5 \mathrm{eV}\right)$. Alternatively ionization of excited sodium via the Penning process is possible:
i) $\mathrm{He}^{*}+\mathrm{Na}^{*} \rightarrow \mathrm{He}^{+}+\mathrm{Na}+\mathrm{e}^{-}$;
ii) $\mathrm{He}^{*}+\mathrm{Na}^{*} \rightarrow \mathrm{Na}^{+}+\mathrm{e}^{-}+\mathrm{He}$

Enhancement of the $\mathrm{Na} \mathrm{D}$ line fluorescence was observed in the presence of the discharge in sodium and hydrogen. This is surprising since studies of the glow discharge indicated consumption of sodium $3^{2} \mathrm{P}$ states and hydride formation and thus an increase in fluorescence intensity is not expected. A possible argument, though it would not support hydride formation, is electronic-vibrational transfer from $\mathrm{Na}$ $3^{2} \mathrm{P}$ to $\mathrm{H}_{2}(\mathrm{v})$ and subsequent vibrational-electronic transfer from $\mathrm{H}_{2}(\mathrm{v})$ if it does not relax before reaching the excitation and observation region. Using the rate constant for the vibrational relaxation of $\mathrm{H}_{2}(\mathrm{v}), \mathrm{v}=3,4$ according to Jennings, ${ }^{17}$ a relaxation time of $2.5 \mathrm{~ms}$ is expected for the hydrogen concentrations used $\left(10^{16} \mathrm{~cm}^{-3}\right)$. This is in excess of the diffusion time of these species through the system indicating that 
$\mathrm{H}_{2}$ (v) does not relax before it has passed through the system. An alternative argument may be a contribution from a chemiluminescent reaction between sodium vapour and excited hydrogen species produced in the discharge. As a result of which excited sodium predominantly in the ${ }^{2} \mathrm{P}$ states is produced, thus increasing the intensity of D-line radiation.

Theoretical quenching curves may be plotted, according to Mitchell and Zemansky, ${ }^{18}$ in which the intensity of radiation $\mathrm{G}\left(\mathrm{kl}, \mathrm{Z}_{\mathrm{Q}}\right)$ emerging from a vapour layer of thickness 1 having an absorption coefficient $\mathrm{k}$ is calculated at various values of $\mathrm{kl}$ (the opacity), for different values of $\mathrm{Z}_{\mathrm{Q}}$ (the number of quenching collisions per lifetime). This enables a quenching curve representative of the specific conditions to be obtained. Calculation of a quenching curve for this particular situation yields good agreement with that produced for hydrogen quenching with an absorbing layer thickness of $1 \mathrm{~mm}$ (Figures 6 and 7).

The experiments carried out indicate the relative abilities of helium and hydrogen to quench the $\mathrm{Na}$ D-line radiation, showing, as expected, the much stronger quenching behaviour of hydrogen. However, the absolute values are undoubtedly influenced by radiation trapping, making calculations of cross-sections inaccurate due to the $\tau_{0}$-factor appearing in the equations used. Calculation of theoretical quenching curves, as outlined by Mitchell and Zemansky, ${ }^{18}$ produces curves in reasonable agreement with those in the present investigations for hydrogen as the foreign gas. Helium exhibits much smaller losses in fluorescence over the same pressure range; this sug-

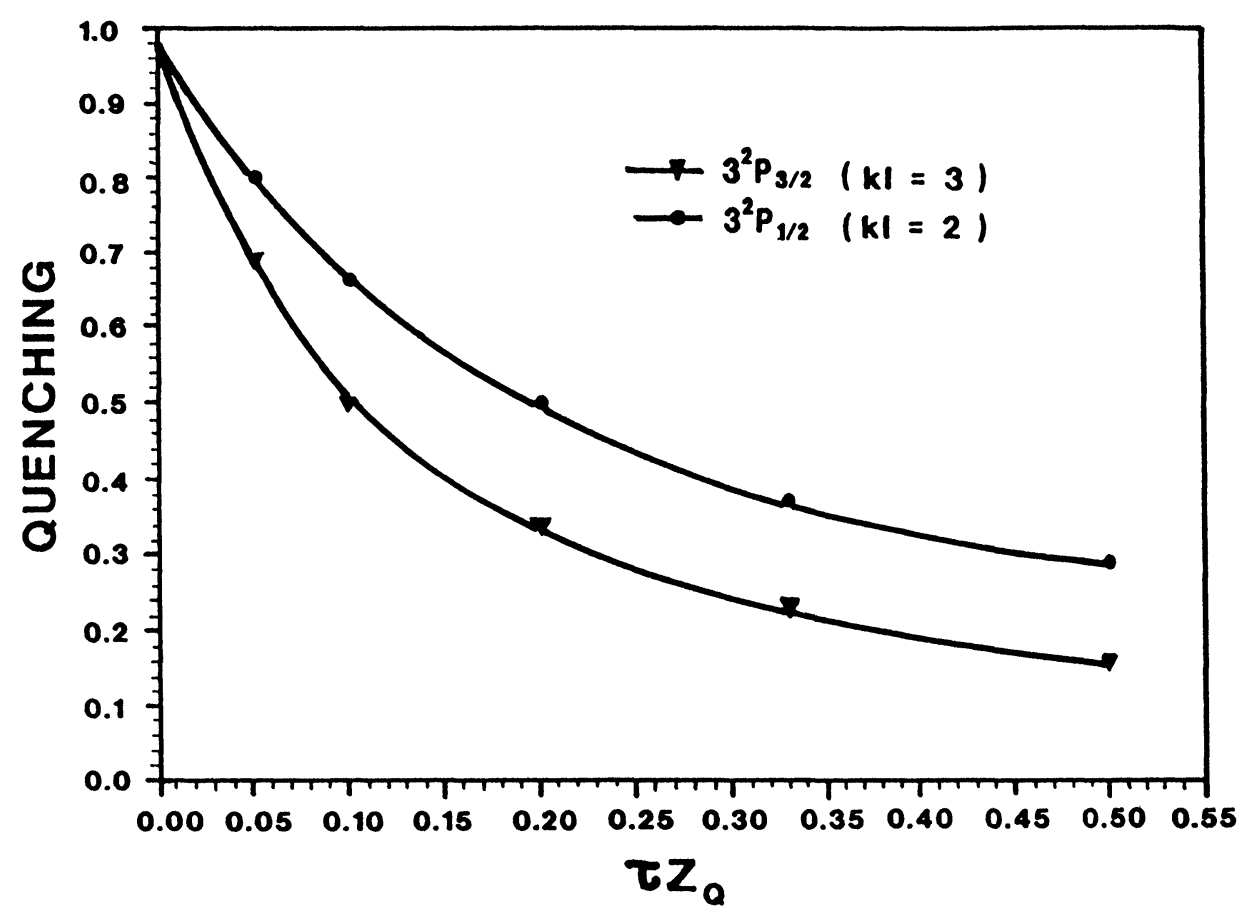

Figure 7 Quenching $\mathrm{Q}$ as a function of $\tau \mathrm{Z}_{\mathrm{Q}}$ for values of $\mathrm{kI}$ corresponding to $\mathrm{Na} 3^{2} \mathrm{P} \frac{1}{2}$ and $3^{2} \mathrm{P} \frac{3}{2}$ 
gests that helium is not a strong quencher of $\mathrm{Na}$ fluorescence. Previous investigations have attributed the decay of sodium resonance fluorescence in the presence of helium to Lorentz broadening of the resonance line by helium. ${ }^{18}$ However, the quenching action of helium is still a somewhat controversial point. ${ }^{19,20} \mathrm{~A}$ possible quenching mechanism is the formation of intermediate quasimolecular $\mathrm{Na}-\mathrm{He}$ pairs. This phenomenon would manifest itself as 'wings' on the D-lines. Although this has been observed at high inert gas pressures ${ }^{21}$ it is not observed in these experiments. However the formation of these species cannot be eliminated since their observation will be dependent on the detection sensitivity. From these experiments it is not possible to be certain whether quenching is present or not, since the quenching curves are dependent on the opacity $\mathrm{kl}$ and larger values of $\mathrm{kl}$ give a curve indicating stronger quenching. This would explain the apparently stronger quenching in the presence of helium than expected.

\section{Lifetime Measurements}

Examples of the signals recorded at helium pressures of $1.5 \mathrm{mbar}$ and $10 \mathrm{mbar}$ are shown in Figure 8. In general one makes the following observation; lifetime of the resonance fluorescence is shorter than that of the off-resonance level e.g. when the $3^{2} \mathrm{P}_{\frac{1}{2}}$ level is excited $\tau_{1}\left(3^{2} \mathrm{P}_{\frac{1}{2}}\right)<\tau_{2}\left(3^{2} \mathrm{P}_{\frac{3}{2}}\right)$. This feature is most noticeable at low foreign gas pressures. This is reasonable since the off resonance level population and its

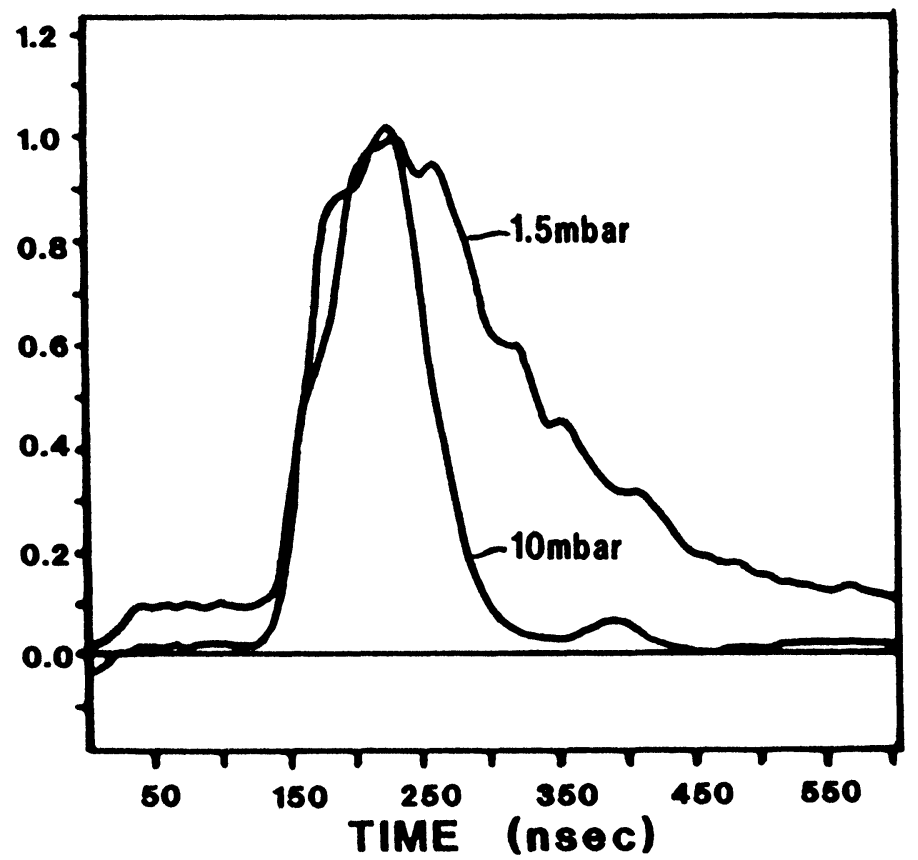

Figure 8 Na $3^{2} \mathrm{P} \frac{3}{2}$ fluorescence decay curves with helium 
subsequent decay are dependent on the collisional transfer from the resonance level. At higher foreign gas pressures the collision frequency is increased and the transfer occurs more rapidly, thereby reducing the difference in the lifetimes of the two levels.

The difference in lifetimes therefore reflects the time taken for the off-resonant level to be populated and subsequently decay. At higher pressures the collision probability is increased and there is virtually no difference between the two levels. Figure $9 a$ and $9 b$ shows this variation of lifetimes of the two fine structure levels with foreign gas pressure. An equilibrium situation is approached in the population of, and the transfer rate between the two levels. Lifetime measurements thus reflect the probability of collisions between foreign gas and excited species and indicate when equilibrium amongst the states is reached. Many of the previous studies of collisions of $3^{2} \mathrm{P}$ states with foreign gas particles use the behaviour of intensity ratios in order to determine when an equilibrium situation is approached. This is dependent on fluctuations in sodium concentration and can yield erroneous results, whereas lifetime measurements are less prone to this problem.

Extrapolating the lifetime data to zero foreign gas pressure yields decay times for the two fine structure levels which are dependent on the transition excited, the foreign gas and in excess of the natural lifetime of $17 \mathrm{~ns} .{ }^{22}$ Kibble et al. ${ }^{12}$ have discussed the

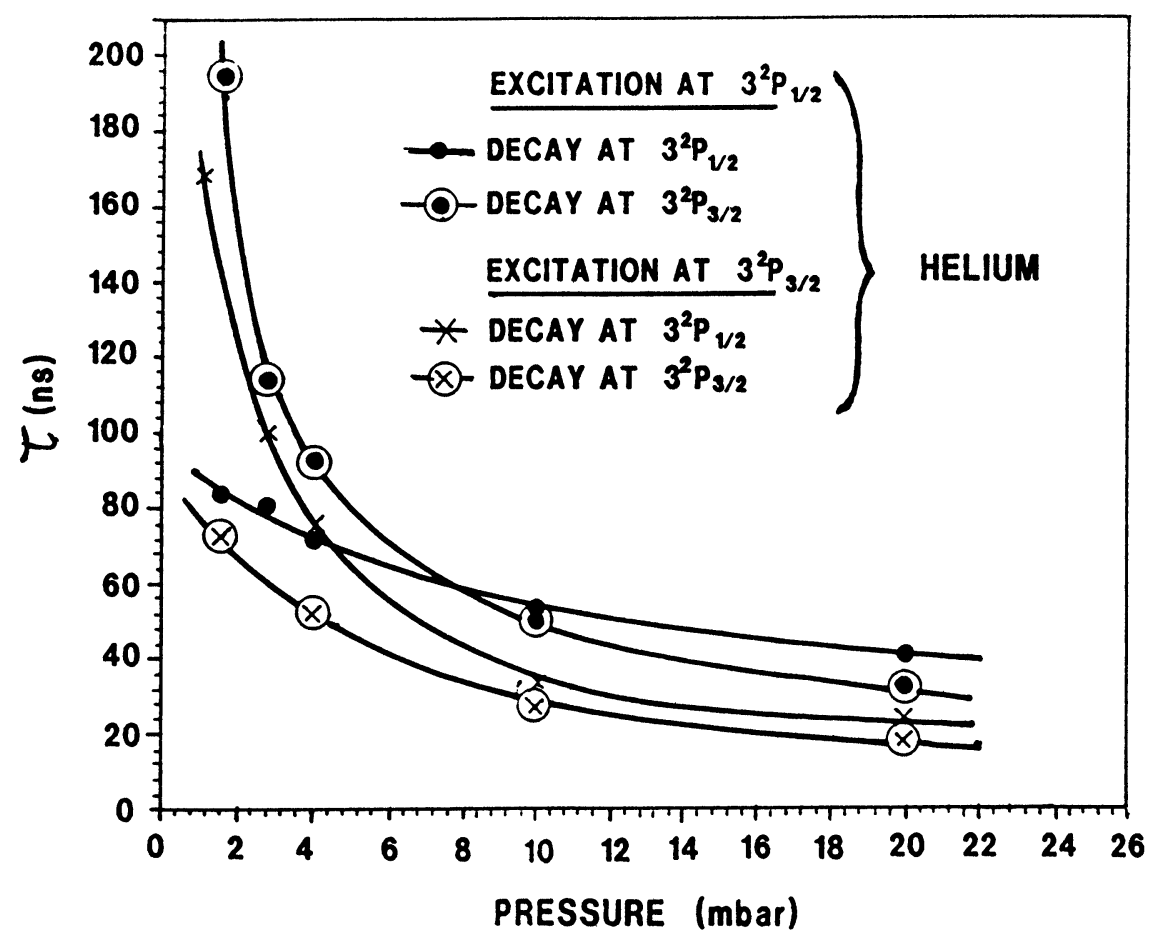

Figure 9a Lifetimes of $\mathrm{Na} 3^{2} \mathrm{P} \frac{1}{2}$ and $3^{2} \mathrm{P} \frac{3}{2}$ versus helium gas pressure 


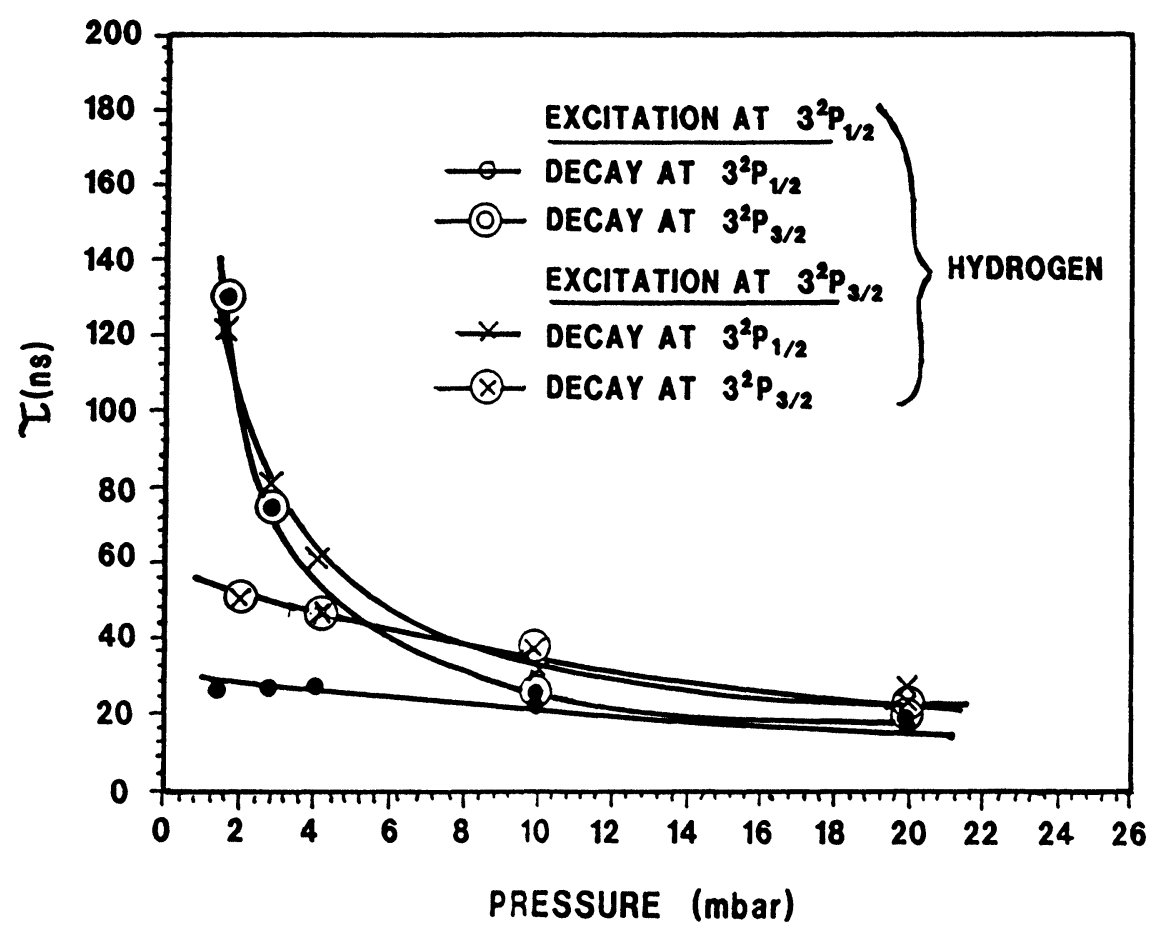

Figure 9b Lifetimes of $\mathrm{Na} 3^{2} \mathrm{P} \frac{1}{2}$ and $3^{2} \mathrm{P} \frac{3}{2}$ versus hydrogen gas pressure

effect of the higher probability of trapping the radiation from the $3^{2} \mathrm{P}_{\frac{3}{2}}$ level on the lifetimes on the fine structure levels. A lifetime of $\sim 200 \mathrm{~ns}$ was measured for the lifetime of the $3^{2} \mathrm{P}$ states at a $\mathrm{Na}$ vapour number density of $3 \times 10^{12} \mathrm{~cm}^{-3}$. At these densities the lifetimes of the two levels could not be separated. In the current investigations the $\mathrm{Na}$ vapour number density is estimated at $\sim 10^{14} \mathrm{~cm}^{-3}$, this density is sufficient to cause, as has been demonstrated in the intensisty measurements, the differential trapping effects on the lifetimes of the two levels.

The slope of the reciprocal lifetime versus pressure plots (see Figure 10) provides information on the collisional quenching cross-sections. Equating the gradients to $\sigma(8 / \pi \mathrm{kmT})^{1 / 2}$ yields cross-section values for quenching for $\mathrm{Na}-\mathrm{He}$ and $\mathrm{Na}-\mathrm{H}_{2}$ collision. These are listed in Table 1. The effects of pressure broadening due to foreign

Table 1 Measured cross-sections for the quenching of $\mathrm{Na} 3^{2} \mathrm{P} \frac{3}{2}$

\begin{tabular}{lc}
\hline Foreign Gas & Cross-section $\left(\AA^{2}\right)$ \\
\hline $\mathrm{He}$ & 8.7 \\
$\mathrm{He}^{*}$ & 11.4 \\
$\mathrm{H}_{2}$ & 12.04 \\
$\mathrm{H}_{2}(\mathrm{v})$ & 6.02 \\
\hline
\end{tabular}


gases was not observed. No curvature in the Stern-Volmer plots (Figure 10) was produced. Although, it should be pointed out that even in cases where pressure
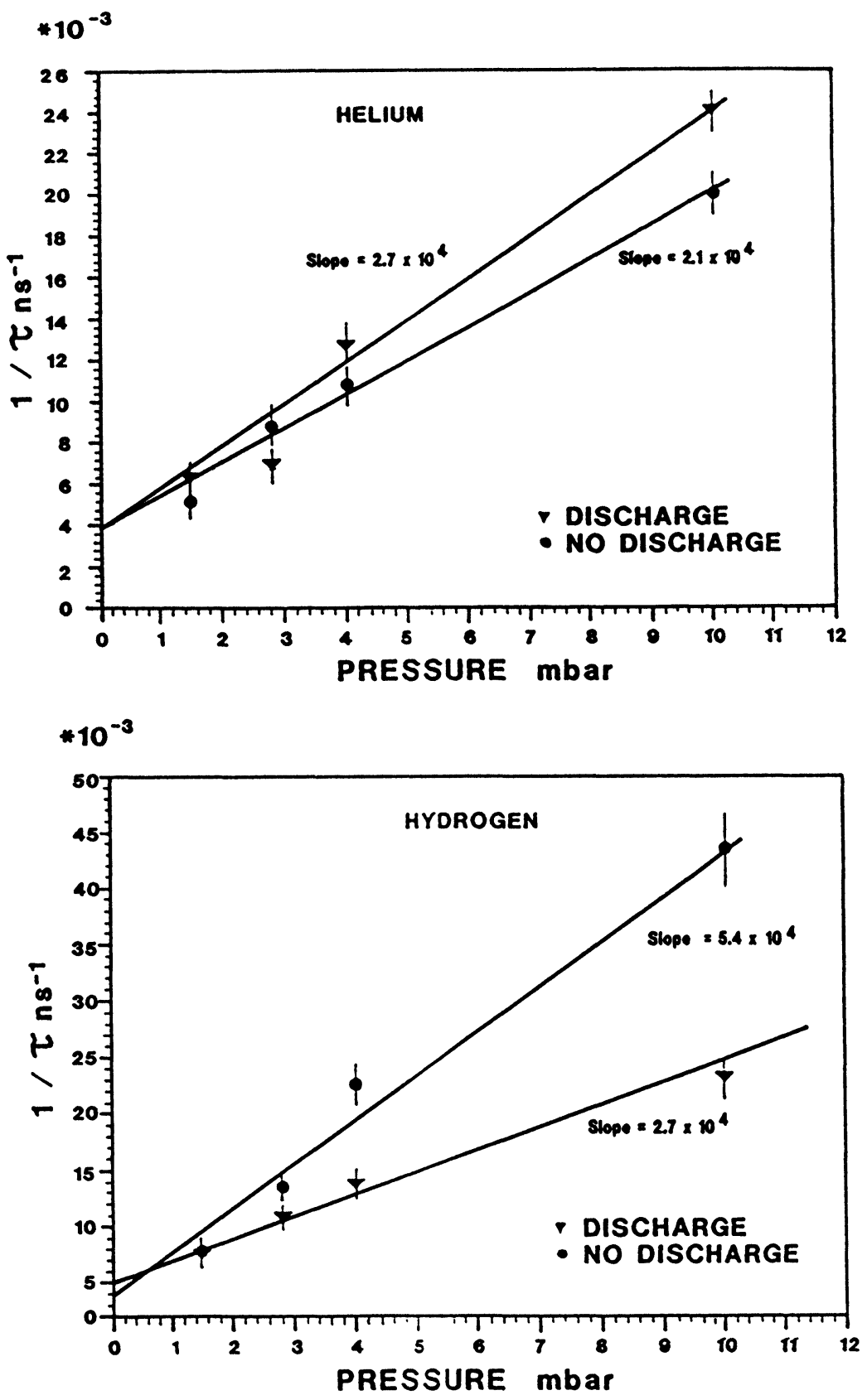

Figure 10 Reciprocal lifetimes of the $3^{2} \mathrm{P} \frac{3}{2}$ level versus gas pressure 
broadening is effective a linear variation can be produced. Quenching cross-section determined by earlier studies are listed in Table 2 .

Table 2 Observed cross-sections for quenching (to the ground states of $\mathrm{Na}\left(3^{2} \mathrm{P}\right)$ ) by hydrogen and helium

\begin{tabular}{lccccc}
\hline Deactivating atom or molecule & \multicolumn{5}{c}{ Cross-section $\left(\AA^{2}\right)$} \\
\hline & $($ a) & $(\text { b })^{8}$ & $(\text { c })^{6}$ & $(\text { d })^{11}$ & $(\text { e })^{9}$ \\
$\mathrm{H}_{2}$ & 23 & - & 9 & 15 & 9 \\
$\mathrm{He}$ & - & 0.34 & 0.3 & - & - \\
\hline
\end{tabular}

Values of the quenching cross-section for helium are a factor of 10 higher than those previously measured. The increased cross-section for collisions with excited helium species supports the findings of the intensity measurements. The quenching cross-section for hydrogen is comparable with those previously measured, which do, however, show a variability of a factor of 2 in some cases. The particular measurement conditions have no doubt contributed to the variability. The larger quenching cross-sections for the excited hydrogen species are not surprising since two quenching mechanisms are possible; transfer to $\mathrm{H}_{2}(\mathrm{v})$ and formation of the hydride.

\section{DISCUSSION}

Whilst the study of the collision processes (1) quenching and (2) interstate energy transfer is not new, the above experiments highlight that the mathematical descriptions used to describe these processes do not satisfy the conditions in these investigations. The treatments outlined earlier are suited only to experimental conditions free of radiation trapping. Furthermore, the process for which the cross-section is sought, is the only process active; there are not competing processes. In these investigations where the conditions are suited to a chemical reaction but where quenching and interstate transfer may also simultaneously occur the earlier approaches are not sufficient.

Consider the quenching process i.e. $\mathrm{Na}^{*}+\mathrm{H}_{2} \rightarrow \mathrm{Na}+\left(\mathrm{H}_{2}\right)^{*}$. Determination of the quenching cross-section from the $\mathrm{I}_{0} / \mathrm{I}_{1}$ vs pressure plot requires use of $\tau_{0}$ which cannot be taken as the natural lifetime if radiation trapping is active. Furthermore, if $\mathrm{Na}$ densities are sufficient for a chemical reaction to occur then the $\mathrm{Na}^{*}$ may become quenched via chemical reaction or by transferring its energy to $\mathrm{H}_{2}$ (v). Thus the quenching cross-section represents both processes. The rate of fluorescence decay is increased when radiation trapping or quenching are effective. Measurements of the decay of fluorescence under radiation trapping conditions with and without the quenching gas present could enable an indication of the contribution of each process.

If the lifetime $\tau_{\mathrm{r}}$ of the fluorescence is obtained firstly at zero foreign gas pressure but with radiation trapping active, and secondly in the presence of the quenching gas to give a reduced lifetime $\tau_{\mathrm{qr}}$ then 


$$
T_{\mathrm{qr}}^{-1}=\tau_{\mathrm{r}}^{\prime-1}+\mathrm{Z}
$$

where $\mathrm{Z}$ is the quenching rate and $\tau_{\mathrm{r}}^{\prime-1}$ is the lifetime due to radiation trapping modified by the presence of the quenching gas.

$$
\tau_{\mathrm{r}}^{\prime-1}=\tau_{\mathrm{r}}^{-1}+\Delta
$$

$\Delta$ is given by $(\lambda v / 4 \pi v)\left(\operatorname{lnk}_{0} R\right)^{-1 / 2}$ where $v$ is the collision frequency, $v$ is the speed of the quenching molecules, $\mathrm{k}_{0}$ is the absorption coefficient and $\mathrm{R}$ is the radius of the tube.

Consideration of the interstate transfer process i.e. $\mathrm{Na}^{*}+\mathrm{He} \rightarrow \mathrm{Na}^{* *}+\mathrm{He}$. Evaluation of the collision transfer frequency and consequently the cross-section for this process utilises the lifetime of the excited states. Under radiation trapping conditions $\tau_{0}$ cannot be given the value of the natural lifetime of the state. If $\tau_{0}=16 \mathrm{~ns}$, $\eta_{1}=0.53$ and $\eta_{2}=1.88$ are inserted into equations (8.1) and (8.2). Collision transfer frequencies $Z_{1}$ and $Z_{2}$ of $5 \times 10^{10} \mathrm{~s}^{-1}$ and $2.7 \times 10^{10} \mathrm{~s}^{-1}$ respectively are produced. If now the values for the parameters $\tau_{0}, \eta_{1}$ and $\eta_{2}$ obtained in these investigations are inserted, then $Z_{1}$ and $Z_{2}$ become $9.5 \times 10^{8} \mathrm{~s}^{-1}$ and $3.4 \times 10^{8} \mathrm{~s}^{-1}$ respectively. Thus the efficiency of the transfer process is reduced under these experimental conditions. $Z_{1}$ is a factor of 50 smaller than its radiation trapping-free value and $Z_{2}$ a factor of 100 smaller, indicating the higher probability of trapping the radiation from the $3^{2} \mathrm{P}_{\frac{3}{2}}$ level. Equations (8.1) and (8.2) should be modified to allow for radiation trapping.

$$
\begin{aligned}
& \mathrm{Z}_{1}=\left(\frac{1}{\tau_{1}}\right)\left(1+\eta_{2}\right) /\left(\frac{1}{\eta_{1}}-\eta_{2}\right) \\
& \mathrm{Z}_{2}=\left(\frac{1}{\tau_{2}}\right)\left(1+\eta_{1}\right) /\left(\frac{1}{\eta_{1}}-\eta_{2}\right)
\end{aligned}
$$

where $\tau_{1}$ and $\tau_{2}$ are the appropriate lifetimes for the conditions.

Although quenching of $\mathrm{D}$-line radiation by $\mathrm{He}$ is a controversial point and the crosssections for it have been small in comparison to diatomic gases, a term may be included to give:

$$
\begin{aligned}
& \mathrm{Z}_{1}=\left(\mathrm{Z}_{\mathrm{Q}}+\frac{1}{\tau_{1}^{\prime}}\right)\left(1+\eta_{2}\right) /\left(\frac{1}{\eta_{1}}-\eta_{2}\right) \\
& \mathrm{Z}_{2}=\left(\mathrm{Z}_{\mathrm{Q}}+\frac{1}{\tau_{2}^{\prime}}\right)\left(1+\eta_{2}\right) /\left(\frac{1}{\eta_{1}}-\eta_{2}\right)
\end{aligned}
$$

If both processes quenching and interstate transfer occur under conditions where radiation trapping occurs, separation of the individual contributions is complicated and cannot be treated by the existing representations of the individual processes.

\section{CONCLUSION}

Whilst many of the features observed in this investigation are to be expected, it should be pointed out that in contrast to virtually all previous investigations of this 
kind which were carried out in the absence of radiation trapping effects this present study includes trapping. It is clearly desirable to study the $\mathrm{Na}-\mathrm{He}$ and $\mathrm{Na}-\mathrm{H}_{2}$ collisional interactions under conditions involving trapping since high $\mathrm{Na}$ densities, responsible for trapping, are required for $\mathrm{NaH}$ formation.

In these investigations where a number of collisional processes may occur simultaneously, the previously developed approaches are not satisfactory. However, the complexity of this system is typical of 'real' reaction dynamics situations.

The interaction between excited helium and hydrogen species is of interest, particularly since the interaction of such species with excited sodium is of paramount importance in the production of the alkali hydride. The increased quenching effect of excited helium species has been explained, however the reduction in quenching cross-section obtained for excited hydrogen is unexpected and needs further study. Investigations of the quenching interactions of these species with sodium is not well documented and further investigation is necessary in order to understand these collisional interactions which can in turn yield an improved understanding of alkali-hydride gas phase formation. The need for a more rigorous examination of collision cross-sections under these very realistic conditions is demonstrated.

\section{Acknowledgements}

This work has been funded Science and Engineering Research Council. The authors also wish to thank Professor C Grey Morgan, and fellow researchers at the Department of Physics, University College of Wales, Swansea.

\section{References}

1. W. Lochte-Holtgreven. Z. Phys., 47, 362, 1928.

2. R. Mankopff. Z. Phys., 36, 315, 1926.

3. J. A. Jordan, P. A. Franken. Phys. Rev., 142, 20, 1965.

4. L. Krause. Appl. Opt., 5, 1375, 1966.

5. J. Pitre, L. Krause, Can. J. Phys., 15, 2671, 1967.

6. D. R. Jenkins. Proc. Roy. Soc. A, 293, 493, 1966.

7. R. G. W. Norrish, W. M. Smith. Proc. Roy. Soc. A, 176, 295, 1941.

8. W. Demtroder. Z. Phys., 166, 42, 1962.

9. H. G. Hanson. J. Chem. Phys., 23, 1391, 1955.

10. B. P. Kibble, G. Copley, L. Krause. Phys. Rev., 159, 11, 1967.

11. H. P. Hooymayers, C. T. T. Alkemade. J. Quant. Spectrosc. Radiat. Transfer 6, 501, 1966.

12. B. P. Kibble, G. Copley, L. Krause. Phys. Rev., 153, 9, 1967.

13. M. I. D'Yakonov, V. I. Perel. Sov. Phys. JETP., 20, 997, 1965.

14. T. Holstein. Phys. Rev., 72, 1212, 1947.

15. J. P. Barrat. J. Phys. Radium 20, 541, 633, 657, 1959.

16. E. A. Milne. J. London Math. Soc., 1, 1, 1926.

17. D. A. Jennings, W. Braun, H. P. Broida. J. Chem. Phys., 59, 4305, 1973.

18. A. C. G. Mitchell, M. W. Zemansky. 'Resonance Radiation and Excited Atoms', Cambridge University Press 1961.

19. E. E. Nikitin, V. K. Bykhovskii. Opt. Spectry., 17, 444, 1964.

20. J. H. Stamper. J. Chem. Phys., 43, 759, 1965.

21. R. E. M. Hedges, D. L. Drummond, A. Gallagher. Phys. Rev. A, 6, 1519, 1972.

22. J. Barker, R. Weston. Jnr. J. Chem. Phys., 65, 1429, 1976. 